\title{
INTERVIEW WITH AIMEE, CHEF DIRECTOR OF TRANSLATORS WITHOUT BORDERS (TWB)
}

Carmen Valero-Garcés

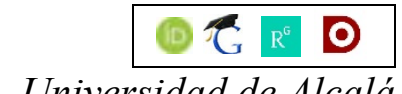

Universidad de Alcalá

carmen.valero@uah.es

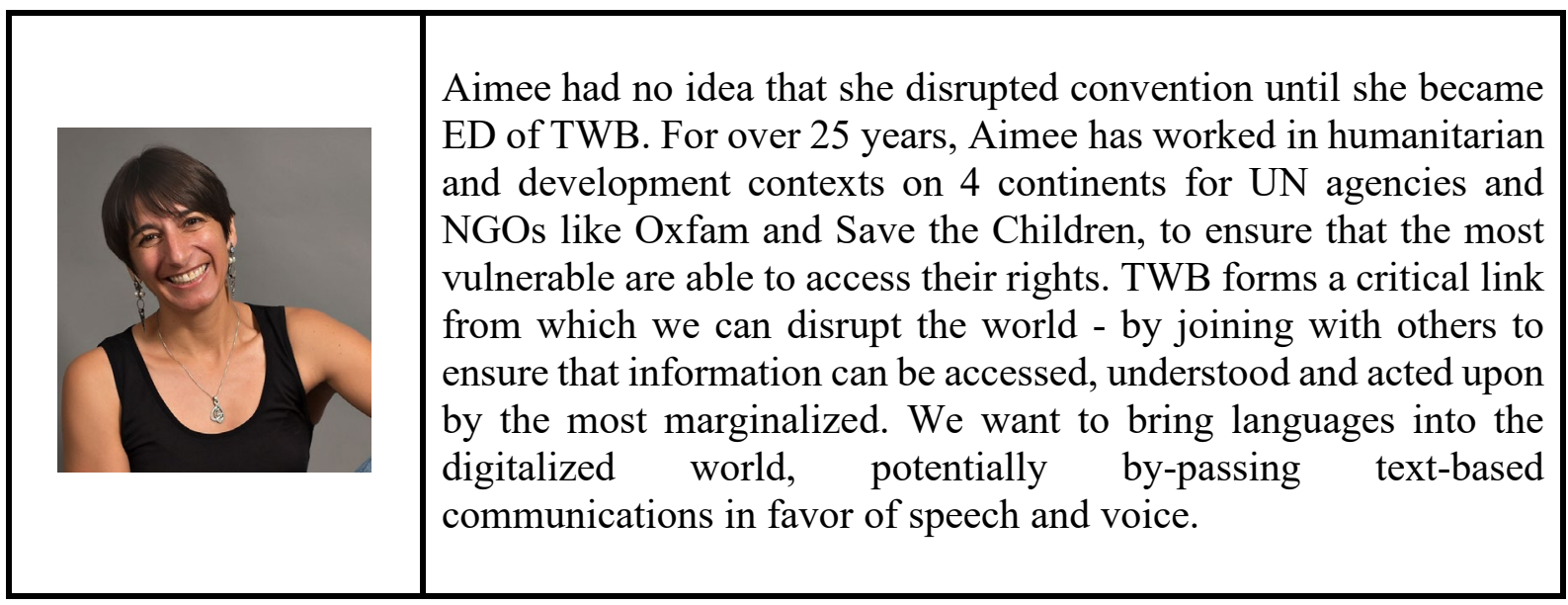

\section{C.V.G. What is TWB? When and why was it founded?}

Originally founded in 1993 in France as Traducteurs sans Frontières by Lori Thicke and Ros Smith-Thomas to link the world's translators to vetted non-profit organizations that focus on health, nutrition and education, Translators without Borders is a U.S. non-profit organization that aims to close the language gaps that hinder critical humanitarian and international development efforts worldwide. TWB recognizes that the effectiveness of any aid program depends on delivering information in the language of the affected population. By maintaining a global network of professional translators, TWB helps non-profit organizations overcome communication barriers, increasing access to critical information and services while fostering a climate of understanding, respect, and dignity in times of great need. Our Vision: A world where knowledge knows no language barriers.

C.V.G. Tell us something about yourself. How did your interest in translation and interpretation come about? And how did you become Executive Director of TWB? 
I have been an aid worker for the past 25 years - working in more than 20 countries. While I speak a number of languages, I was constantly needing others to translate or interpret for me. Sometimes, these were teachers in small villages. Many spoke English well, but none were trained to be interpreters or translators. Some of the people I worked with who were from the countries in which I worked would also translate or interpret. I often found myself "schooling" them in the do's and don'ts of interpretation. And I was never sure of the accuracy of the interpretation or translations.

When I left South Sudan in 2015, having worked in a number of brutal civil wars and devastating natural disasters, I wanted to do something beyond providing aid or technical assistance. The people in those countries really needed opportunity and information. They needed to be able to communicate and to get the information they wanted and not what aid agencies thought they needed. When I saw the position at TWB, I saw the potential the organization could have to facilitate people to get the information they need and want, when they need it and want it, in a language and format they understand. So, I applied and was hired. It's the best job I've ever had.

\section{C.V.G. Nowadays, how many people collaborate and how many languages does TWB deal with?}

$\mathrm{Oh}$ - that's a hard question. The number of languages is over 200, but counting languages isn't an exact science. The difference between a language and dialect isn't well defined.

How many people? We say TWB's community includes over 30,000 linguists. The number of people who have translated for TWB is actually much larger - and it varies. Translators might have time to take on a TWB job in May, but not in April or June. 30,000 is the approximate number of people who volunteer for TWB in a year. And we always need more - in every pair.

\section{C.V.G. TWB acts in situations of great danger or emergency situations. Are there specific lines of actions for TWB? War? Racial conflict? Religious conflict? Gender conflict? How does TWB decide to act?}

Yes, we often respond to crises and I'll talk about that below. I think it's important, though, to recognize that people need information they can understand every day. The bulk of the 20 million words translated every year are for organizations who are not responding to crises, and are, every day, all the time, supporting people. For example, we work with the Free Wheelchair Mission, an organization that provides wheelchairs to people in need. They work all over the world, in some very remote communities. We translate information on care and maintenance of the wheelchairs. This is super important because there may be no one in those communities who has seen a wheelchair before or who can help repair them.

We also work with SmileTrain, an organization that does cleft palate surgery. We translate information for the children and their families on the process and recovery.

TWB current works with about 150 organizations, working all over the world in over 200 languages.

\section{C.V.G. When TWB decides to intervene or develop a translation campaign, what protocol(s) are followed?}

In general, we respond when communications between aid workers and those in needs are hampered, often by language.

When there is a crisis, we first ask if there's anything we can add to work that others are doing. There's no point in providing services if no one will use them.

We will generally try to find out what languages are spoken in the area affected. You might think this is easy, but it's incredibly difficult. There is no openly available credible 
resource with that information globally. Some countries will have census or other information that can help. But, this is usually not mapped out clearly for responders. TWB has a project dedicated just to language mapping.

You might be surprised to learn that language hasn't been a consideration in humanitarian response. Even in the UK and US, it's rarely considered. We've done maps, for example, of the languages spoken in the western US to inform responders what languages were needed.

If we see a need for our support, then the first thing we often do is a language map - and then work with data collection organizations to improve our understanding of the languages used.

We will then put out a call for translators globally. Usually our main partners will start requesting translations very quickly; others will need to be convinced that language is an issue. If we decide that we need to have teams in country, then we will also send one or two of our experienced humanitarian responders to the area.

Usually, we will do rapid research on the language and communications issues. TWB has done some ground-breaking work on the differences between Rohingya and Chittagonian, on language issues in the Ebola response in eastern DRC, and on how language can support humanitarian efforts in Mozambique. Based on this work we will start working with local and global linguists and advocating for the importance of language.

While doing this, we will start translating information, developing glossaries, and training field interpreters. We will also start understanding the context better - are the languages written, how do people prefer to give and receive information, what sources do they trust, are phones widely available and do men and women have equal access to them, what are literacy levels, how to people understand signs - with others, we work quickly to listen to people and make sure that they get the information they want in a way that is useful to them. We have recently started developing localized posters, doing more subtitling of videos, and working with radio stations to ensure that information is disseminated.

And, going further, we are transcribing and translating people's feedback, developing tools so that people can make their needs and concerns heard effectively, and innovating language technology to really listen to people, be it basic voice recognition systems or chatbots - so people can ask questions and get answers.

We've responded to many, many crises - the Nepal earthquake, the Ebola outbreak in West Africa and then the 3 outbreaks in DRC, we responded in Greece, in Bangladesh, in Mozambique, in Haiti and to many fires, floods, and earthquakes. There are always people who need information in their language. Most recently, we have started supporting organizations responding to refugees from Tigray.

\section{C.V.G. How has the COVID-19 pandemic affected TWB? What actions are you taking to help with LLD?}

TWB has always been a dispersed organization. We have always worked from home, so COVID-19 has had limited impact on our operations. Our teams in Nigeria, Bangladesh, and DRC had to scale down face to face training, but, because we were always dispersed, they already had the tools to start remote work themselves.

Our response to the COVID-19 pandemic has been multi-faceted. Our two biggest pieces have been our COVID-19 glossary, currently over 50 languages, and the TICO-19 project, where we led a consortium to develop COVID specific language data in 30 languages. The consortium included the major tech companies and many large LSPs and universities.

\section{C.V.G. Do governments and public and/or private institutions collaborate actively with TWB when required? How?}


Yes. We work very closely with mostly non-governmental organizations, including some of the largest, like UNICEF, Save the Children, World Vision, and Oxfam. And in collaboration with governments. For example, we are supporting the Ministry of Health in DRC with the first multilingual chatbot using natural language understanding in Congolese Swahili and Lingala.

C.V.G. Coming back to translation and translators, how can a translator become a member or collaborate with TWB? What are the main requirements?

You just have to sign up! You can volunteer with TWB if you are fluent in at least one language other than your native language. Whether you are interested in translating medical texts or translating for crisis response, there are engaging projects available to suit all preferences. Professional translators are especially encouraged to apply.

C.V.G. Considering that TWB also translates into languages of lesser diffusion (LLD) when training is not generally available, what profile do T/Is working in LLD usually have? Local ad hoc? Untrained? Professional? Male? Female?

Almost everywhere there are people who are multi-lingual. We do a lot of skills building and training, particularly on CAT tools, for those who are new to them.

\section{C.V.G. What about translation quality assessment? How is a translation evaluated? What criteria are applied?}

We have a revision process. In general, we use some QA tools and Taus' DQF.

C.V.G The theme of this issue of FITISPOs IJ $(\mathrm{vol} 8,2021)$ is the human factor. However, considering the influence of machine translation and artificial intelligence in the translation industry, what impact is this having on the translators and in their product? How does it affect the majority languages and the LLD?

TWB is working to develop better language tool for speakers (and translators) of marginalized languages. Right now, there isn't much out there - and there aren't many translators out there. And, there isn't much demand for them.

TWB builds awareness of the importance of information in the right language, we support organizations to ensure that critical information is communicated in the languages people use. And, to do that, we train translators and interpreters and develop language tools.

\section{C.V.G Would you say that English is becoming a lingua franca? What are the main languages of use?}

In many of the places that TWB works, the lingua franca isn't English - it's Swahili or Hausa or Bangla, for example. Our research has found that those who are least likely to speak the lingua franca of the region are those who are most vulnerable and most in need of information - women, children, the elderly, the disabled.

\section{C.V.G In which countries does TWB have the greatest presence?}

Right now, we have country offices in Nigeria, Bangladesh, and Nigeria. TWB also has tremendous language communities in French, Spanish, Arabic, Swahili, and many others. We have an amazing Language Associate program, our program to appoint volunteer community members to support community engagement activities, as well as the development of key language resources such as style guides and terminology, and quality assurance support. We recently onboarded our new Amharic Language Associate, in addition to our Language Associates for French, Spanish, Somali, and a few other. And expect to soon have Oromo, Hindi and Pashto Language Associates on board. 


\section{C.V.G Could you explain a case/example that has particularly affected you?}

There are so many! When we were demonstrated a language tool, one person in Nigeria told us that he didn't know his language was special enough to put on a computer.

On a personal level, the effect that TWB's work has on people never ceases to amaze me. As I mentioned, I've been working all over the world for over 25 years and this is the first time that people - in villages or in high level UN meetings - tell me how much they appreciate what we do. They tell me that TWB helps in ways that no other organization does, by prioritizing their culture and their language. It makes them proud that their language is "important enough;" they say that TWB really wants to listen and understand because we respect their language and ask them to express themselves in the language and ways that they are most comfortable. We don't ask them to understand our language and our ways. I've never had people who don't know me just want to thank me for what TWB does.

\section{C.V.G Has TWB received any recognition, or is there any progress in its recognition?}

We've received a lot of recognition. Thanks to our global community linguists, TWB's work is well-recognized. We've won innovator awards, and had many, many articles published about our work. Our work has been highlighted in The Economist, Forbes, Voice of America, WIRED, and many others.

TWB has Special Consultative Status at the UN. We are also registered as a not for profit (501c3) in the US and a Charity in Ireland.

\section{C.V.G Do you have any recommendations for academics interested in training and research in this area? And for practitioners?}

If there are academics who are interested in doing applied research, please do get in touch. TWB has a small, but mighty, research team. We are currently looking for funding to do (broadly) research on language and behavior change (does information in the right language and format help people better understand the benefits and risks of COVID vaccines, for example) and language and trust building (if health care workers use local languages, are they more likely to be trusted by their community?).

\section{Date of reception/Fecha de recepción: 25/01/2021 \\ Date of acceptance/Fecha de aceptación: 31/01/2021}

How to cite this article?/ ¿Cómo citar este artículo?

Valero-Garcés, C. (2021). Interview with Aimee, Chef Director of Translators Without $\begin{array}{lllll}\text { Borders } \quad \text { (TWB). FITISPos-International Journal, } & \text { 8(1), }\end{array}$ https://doi.org/10.37536/FITISPos-IJ.2021.8.1.294 